\title{
Analysis of Facet Temperature Distribution of Semiconductor Lasers
}

\author{
Tiansheng ZHAO, Zaijin LI*, Te LI, Peng LU, Yi QU, Baoxue BO, Guojun LIU, \\ Xiaohui MA, Yong WANG
}

National Key Lab on High Power Semiconductor Lasers, Changchun University of Science and Technology, Changchun 130022, China

* lizaijin@126.com

Keywords: Semiconductor laser; COMD; Temperature; ANSYS

\begin{abstract}
In this paper catastrophic optical mirror damage(COMD) mechanism of the semiconductor laser is analyzed. COMD is one of major device damage mechanisms, which is drastically limited laser lifetime and output optical power. The theoretical model that based on heat source with injection current and optical power to describe the temperature distribution of laser is builded. Through analyzing the edge-emitting semiconductor lasers by using ANSYS, we can describe change of facet temperature distribution at COMD events. the results of simulation show that the main reason of COMD is oxidation of the semiconductor laser in facet which caused by optical absorption.
\end{abstract}

\section{Introduction}

Nowadays, high-power semiconductor lasers have been widely used in the broad field of science. It is important that the reliability of lasers. COMD process is one of the major mechanisms, which drastically limits laser lifetime and emits optical output power. So, its emphasis of study is how to avoiding the COMD of device-cavity surface. For the majority of high power laser, COMD phenomenon of the facet surface induces high optical power density which limits the maximum power of the device, and affects the reliability of the device lifetime [1]. So, in the beginning of the theoretical design of devices, it should analysis simulation of facet temperature to determine the COD threshold of the device. Especially, it is important that improves the reliability of high power lasers. It is necessary that simulation of facet temperature distribution. And it also provides a reference which can improve process of cavity surface and the COMD threshold.

Semiconductor lasers are basically made of a gain section between two facets. When a laser is driven into higher output powers, the facet regions absorb light, hence making the facets hot, and provoking a "thermal runaway" system and eventually irreversible laser destruction [2]. It is so called catastrophic optical damage COMD, and it is characterized by a sudden drop in the output power at a certain maximum injection current.

\section{Principle of COMD}

COMD is a kind of cavity surface damage degradation. On one hand, the quantum well which is typical structure of edge-emitting laser, is by internal biaxial compressive strain in the strained quantum well laser facet into a compressive strain. It is resulting in the end at the band gap decreases, increasing the absorption of light; on the other hand, the semiconductor laser cavity surface at the existence of surface states and interface states. These are non-radiative recombination centers, and their presence will lead to light absorption and increase the cavity surface temperature. When the electromagnetic radiation created by the electric field strength exceeds a critical value, corresponding to the radiation power density exceeds a certain threshold, high energy density optical make micro-cavity surface-melting and decomposition. Near the surface of the optical absorption, the surface recombination current density increases, the local is a lot of heat. With the temperature increasing, the absorption coefficient increased to become a vicious circle [3]. 
Among them, the band gap of the material and the laser damage threshold is closely related to the general band gap the greater the higher the damage threshold; it has a positive correlation for most materials, melting point, mechanical strength, and damage threshold.

The results show that the main reason of the semiconductor laser to produce catastrophic optical damage is the oxidation of the semiconductor laser cavity surface. Inhibit the oxidation of the surface of semiconductor laser cavity can improve the laser damage threshold of optical thin films, semiconductor laser output power and device reliability. For the uncoated laser facet, the triggering of these loops are formed due to the end surface state of causing non-radiative recombination and the cavity surface temperature rises, the absorption coefficient increases. Facet coating, although the make COD power higher than the coating level, but also have a non-radiative recombination due to the presence of interface states between the film and laser materials, and thus the same will also appear COD. Laser facet oxidation is suppressed, but the high power density, high energy release at the interface will accelerate the proliferation of the interface defects .

This can be explained as follows; before COMD, the optical power density in the cavity is very high and with it the light absorption and temperature near the output facet region. COMD is triggered at the point on the facet where temperature is highest, causing massive damage (and melting) near the output facet were COMD starts. This causes a chain reaction and a gradual defects growth. With increasing defects density in the cavity, the optical power density decreases and it light absorption and temperature. Finally the optical power density becomes too low to cause further damage; hence no further defects can grow anymore.

\section{Ansys Simulation}

The transverse structure of the device, material parameters and the distribution of heat sources of the laser used for ANSYS are presented in Table 1 [4-5].

Table 1 ANSYS simulation of structural parameters and material parameters

\begin{tabular}{|c|c|c|c|c|}
\hline Layer & $\begin{array}{c}\text { Thickness }[\mu \\
\mathrm{m}]\end{array}$ & $\lambda[\mathrm{W} /(\mathrm{mK})]$ & $\mathrm{Ch}[\mathrm{J} /(\mathrm{kgK})]$ & $\rho[\mathrm{kg} / \mathrm{m} 3]$ \\
\hline substrate & 100 & 44 & 327 & 5318 \\
\hline Al0.6Ga0.4As(n-cladding) & 1.5 & 11.4 & 402 & 4384 \\
\hline Al0.6Ga0.4As(waveguide) & 0.35 & 11.1 & 378 & 4696 \\
\hline active layer & 0.007 & 44 & 327 & 5318 \\
\hline Al0.6Ga0.4As(waveguide) & 0.59 & 11.1 & 378 & 4696 \\
\hline Al0.6Ga0.4As(p-cladding) & 1.5 & 11.4 & 402 & 4384 \\
\hline GaAs(cap) & 0.2 & 44 & 327 & 5318 \\
\hline p-contact & 1 & 318 & 128 & 19300 \\
\hline In(solder) & 1 & 82 & 230 & 730 \\
\hline
\end{tabular}

According the references, we suppose the ambient temperature is taken $300 \mathrm{~K}$, p-n junction voltage drop is taken $1.97 \mathrm{~V}$. The injection current is set $0.16 \mathrm{~A}$. The threshold current of laser is $0.5 \mathrm{~A}$. Spontaneous radiation quantum efficiency of laser is 0.66 , internal quantum efficiency of stimulated emission is 0.9 , spontaneous emission photon escape factor from the active region is 0.4 , and the emitting efficiency is 0.6. Convection exchange coefficient is taken as $35 \times \mathrm{WK}^{-1} \mathrm{~m}^{-2}$ [6].

According to the experimental experience and the references, we select the following loading boundary conditions. Based on experimental experience, we recognize COMD event is happened between 2A to 2.05A. So, we select 2.02A as the threshold of the COMD injection current. We can get thermal runaway obviously at 2.5A of injection current. So, the following boundary and initial conditions have been assumed. Boundary conditions under steady state conditions[7], the injection current is taken $0.5 \mathrm{~A}$. The threshold of the COMD boundary conditions, the injection current is $2.02 \mathrm{~A}$. When the temperature gets thermal runaway, the injection current is $2.5 \mathrm{~A}$ 


\section{Result and discussion}

We can get the boundary conditions under steady state conditions: the injection current is taken $0.5 \mathrm{~A}$. Just like the figure shows that the heat is mainly focus on active region. When the laser is emitting, the corresponding temperature increases in the laser active region are of $2.21 \mathrm{~K}$.

As the injection current increasing, the heat spread, and the temperature increased. High temperature leads cavity surface to oxidation. After COMD event, we can see from Fig.1 that heat of facet distributed in wider areas. The facet temperature increases in the laser active region are of $100 \mathrm{~K}$.

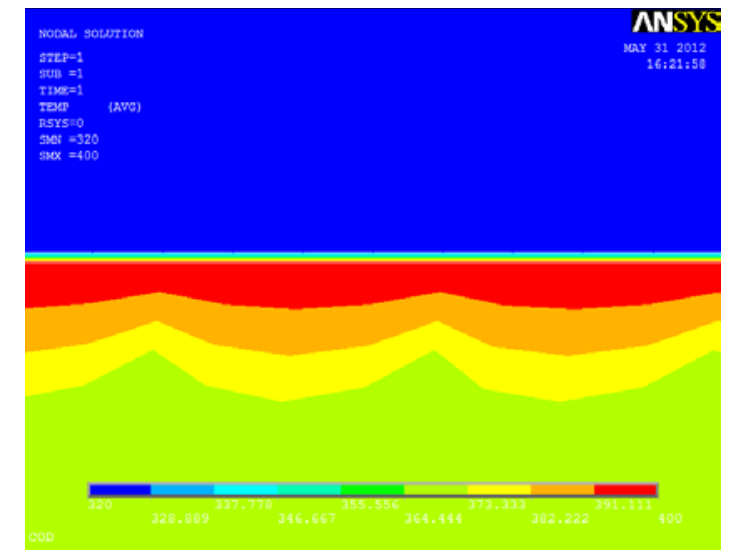

Fig.1 schematic diagram of temperature field distribution after COD event

Laser injection current to reach the threshold current, light-emitting lasers start lasing, the laser output power with injection current increases, the cavity surface temperature and increased with the injection current increases, and finally to the COMD threshold.

Figure.2 shows temperature of laser that increase with injection current increasing. After the COMD event, results in lower light absorption and non-radiative recombination at the facet, leading to a temperature decrease.

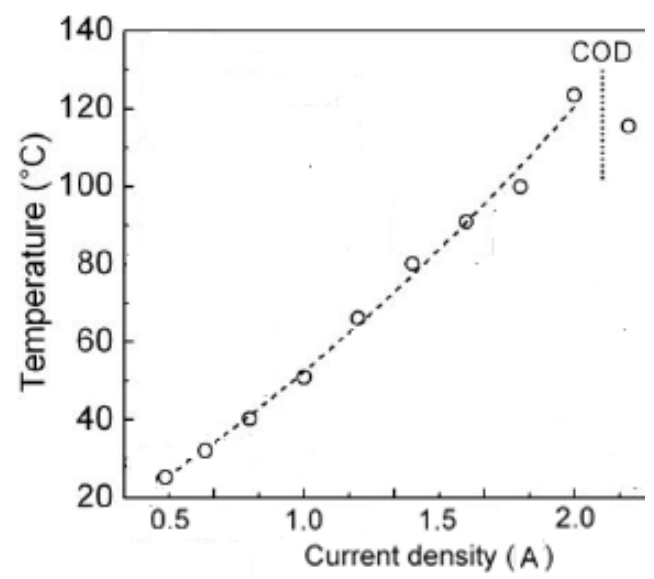

Fig.2 relationship of injection

Fig. 2 shows temperature of the same lasers versus current before COMD. The linear increase of temperature without indicates that the facet temperature increase is mainly due to absorption of laser light at the output facet. After COMD, the sudden drop in power results in lower light absorption at the facet, leading to a temperature decrease.

Through simulation, we can see that the temperature is gradually increased, when the work of the center of the active region of semiconductor devices, especially in the quantum well layer, its melting point is limited and high-temperature made cavity surface oxidation. So there is bound to the occurrence of COMD. With the increase of output power, corresponding to the temperature 
increases, and eventually reaches the damage threshold.

\section{Conclusion}

In summary, we analysis simulation of facet temperature in semiconductor lasers by ANSYS, and discuss the reason of COMD. Through simulation, we describe the change of facet temperature distribution before and after the COMD event. It is intuitive and clear-cut that the simulation of theory, at the same time, it is easy to measure in the analysis.

\section{Acknowledgement}

In this paper, the research was sponsored by NSAF (NO. U1330136), the National Natural Science Foundation of China (NO. 61107054), National Key Laboratory Foundation (233360) and the Jilin Province Science and Technology Development Plan Item (NO. 201201124).

\section{References}

[1] G. Chen and C. L. Tien , "Facet heating of quantum well lasers,” J. Appl. Phys.74 (4), pp. 7054-7060, 15 August 1993.

[2] M. Bou Sanayeh, P. Bricka, W. Schmida.et, "The physics of catastrophic optical damage in high-power AlGaInP laser diodes,” Proc. of SPIE Vol. 6997, pp. 6997031-12, 2008.

[3] Jens W Tomm, Mathias Ziegler, "New approaches towards the understanding of the catastrophic optical damage process in in-plane diode lasers," Proc. of SPIE Vol. 7230, pp. 72300V1-9, 2009.

[4] Pengcheng Lu, Bifeng Cui, Weiling Guo.et, “Thermal characteristics simulation of semiconductor lasers based on ANSYS," Semiconductor Technology Vol. 29 No. 4, pp. 45-51, April 2004.

[5] Michal Szymanski, "Mathematical Models of Heat Flow in Edge-Emitting Semiconductor Lasers,” Institute of Electron Technology, pp. 3-28, 2009.

[6] Jan Pomplun, Sven Burger, "Finite element simulation of the optical modes of semiconductor lasers,” Phys. Status Solidi B 247, No. 4, pp. 846-853, 2010.

[7] E.Kowalczyk, L.Ornoch, Z.Gniazdowski.et,"Dynamics of thermo-optical properties of semiconductor lasers,” Proc. of SPIE Vol. 6456, pp. 64561G1-9, 2007. 